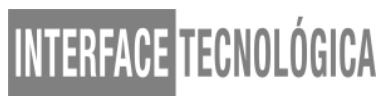

\title{
UM ESTUDO SOBRE GESTÃO PELA QUALIDADE TOTAL NA INDÚSTRIA DE MÁQUINAS E EQUIPAMENTOS
}

\author{
A STUDY ON TOTAL QUALITY MANAGEMENT IN THE MACHINERY AND \\ EQUIPMENT INDUSTRY
}

Leandro Rogério Gonçalves - goncalveslds@gmail.com

Angelita Moutin Segoria Gasparotto - angelita.gasparotto@ fatectq.edu.br

Faculdade de Tecnologia de Taquaritinga (FATEC) -SP -Brasil

DOI: 10.31510/infa.v16i2.636

\section{RESUMO}

No cenário econômico mundial, melhoria contínua é um assunto muito abordado e extremamente necessário, as empresas que produzem máquinas e equipamentos estão aumentando consideravelmente sua competitividade aplicando o conceito de qualidade total, onde atender as necessidades dos clientes é um fator muito importante na sobrevivência competitiva da empresa. Neste ambiente, a concorrência por melhorias vem alcançando padrões de eficiência muito elevados em relação às metodologias de qualidade. Uma organização que pretende permanecer no mercado, a qualidade total e melhoria contínua são necessidades cada vez mais cobradas quando se trata de concorrência industrial, há órgãos regulamentadores que auxilia e cobra adequações para que padrões e normas vigentes sejam respeitados, é imprescindível que as organizações sejam certificadas. Tais órgãos têm a competência de manter estes padrões, pois sugerem melhorias dos processos e produtos atendendo seus requisitos. $\mathrm{O}$ artigo mostra as etapas de implantação em uma indústria de maquinas e equipamentos, seus processos em meio à metodologia aplicada e seus desafios. Cabe salientar que os objetivos da Qualidade total seguem seus padrões em todo o fluxo operacional, desde o fornecimento de matérias primas até o produto acabado para os setores de expedição e logística.

Palavras-chave: Indústria de maquinas e equipamentos, qualidade total, melhoria contínua.

\begin{abstract}
In the world economic sense, continuous improvement is a much-discussed and extremely necessary subject, companies that produce machines and equipment are increasing their competitiveness considerably applying the concept of total quality, where meeting the needs of customers is a very important factor in the competitive survival of the company. In this environment, competition for improvements has been achieving very high efficiency standards in relation to quality methodologies. An organization that intends to remain in the market, total quality and continuous improvement are increasingly demanded when it comes to industrial competition, there are regulatory agencies that assists and charges adjustments so that standards and current standards are respected, it is imperative that organizations are certified. These bodies have the competence to maintain these standards, as they suggest improvements in
\end{abstract}




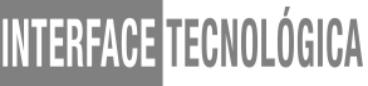

processes and products that meet their requirements. The article shows the implementation stages in an industry of machines and equipment, its processes in the midst of applied methodology and its challenges. It should be noted that the objectives of Total Quality follow its standards throughout the operational flow, from the supply of raw materials to the finished product for the shipping and logistics sectors.

Keywords: Machinery and equipment industry, total quality, continuous improvement.

\section{INTRODUÇÃO}

A procura por resultados e melhoria continua na indústria metal mecânica, fez com que o segmento crescesse exigindo a implantação de novas técnicas de qualidade para suprir os setores e as necessidades nas organizações.

Esse assunto é de extrema importância para o desenvolvimento contínuo das organizações, a qualidade de produtos e serviços é vital onde o senário da concorrência se torna cada vez mais acirrado. A busca pela excelência na qualidade, é um diferencial competitivo das empresas nos dias atuais.

De acordo com Martinez-Costa (2013), uma nova forma de abordar a qualidade tem como um todo procurar atingir objetivos visando a melhoria da qualidade em todos os processos organizacionais.

Assim, o objetivo geral desta pesquisa é apresentar um estudo sobre os métodos de gestão pela qualidade total que será de fundamental importância na busca pela excelência de produtos e serviços garantindo a competitividade das empresas, os resultados obtidos contribuirão no desenvolvimento do relacionamento entre cultura organizacional e gestão pela qualidade total, entendendo a influência positiva cultural dentro da gestão. Visto que a importância do artigo é apresentar um estudo abordando a necessidade pela busca da qualidade total na fabricação de máquinas e equipamentos.

O método proposto no estudo foi por meio de artigo, propondo melhorias nos setores de fabricação de maquinas, equipamentos e informações relevantes sobre o assunto proposto. Entretanto o conteúdo de pesquisa usado para desenvolver esse trabalho foi o de artigo científico. $\mathrm{O}$ artigo será feito em cinco seções, que retratam o estudo proposto com um todo.

O artigo será dividido e estruturado incialmente com a introdução, com o início do tema, será mostrado as consequências positivas na busca da gestão pela qualidade total, onde a 
implantação de métodos buscando melhorias propostas de qualidade atingindo resultados favoráveis e conclusões obtidas através do estudo.

\section{O ESTUDO SOBRE GESTÃO PELA QUALIDADE TOTAL NA INDÚSTRIA DE MÁQUINAS E EQUIPAMENTOS.}

O estudo é de total importância na área metalúrgica, mecânica e da qualidade, tendo em vista que os processos na qual são submetidos os componentes e os colaboradores, não atinge o objetivo sem o mínimo de requisitos envolvendo a qualidade em cada etapa do processo, onde é essencial o comprometimento de toda a equipe.

Tendo como referência os estudos dos maiores nomes conhecedores do assunto como, Dahlgaard, 2013; Green, 2012; Martinez-Costa, 2013; Gambi. L. N, 2015; Haffar. M 2013, entre outros, conclui-se que, segundo Castro, A et al. (2007), para implantação e melhoria contínua da qualidade total é necessário geri-la. Gestão da qualidade total, são comportamentos estratégicos que antecipem o agir e o produzir, conta com a mudança de postura organizacional e gerencial com o objetivo de atingir o sucesso de uma empresa, além da satisfação dos clientes, a qualidade total também procura a satisfação dos stakeholders, que são peças importantes interessadas na busca da excelência e no sucesso da organização.

Gerir a qualidade total é de extrema importância o compromisso das pessoas de cada nível hierárquico objetivando a eficiência e a valorização do cliente, em uma abordagem mais ampla, tornar a empresa mais competitiva e eficaz planejando e compreendendo cada processo responsável pela conclusão do produto. De acordo com Delic et al (2014), existe inúmeros aspectos que motivam a gestão pela qualidade total, treinamento e desenvolvimento dos colaboradores, atender/exceder aos requisitos dos clientes; defende Rachid (2013) o envolvimento dos trabalhadores, melhorar a imagem empresarial; ganho de mercado; melhorar o moral de sua equipe de colaboradores; melhorias de processos, produtos e serviços. Sendo assim, a gestão da qualidade sugere uma nova abordagem gerencial gerando a necessidade de uma nova cultura organizacional, defende Schein (2009), “A cultura está para o grupo assim como a personalidade ou caráter está para o indivíduo”. 


\subsection{Controle de qualidade total (TQC)}

O TQC ou Total Quality Control (Controle de Qualidade Total), trata-se de um conceito de gestão da qualidade que objetiva transcender o conceito de qualidade aplicada no produto, pois visa não apenas atender o cliente, mas todos os interessados (stakeholders).

Para entender o princípio ao controle da qualidade referia-se ao enquadramento dos produtos e serviços buscando a garantia de suas exigências técnicas, sendo assim resumia-se a qualidade total na ausência de não conformidades no produto acabado, sendo mais tarde controlado estatisticamente, permitiu-se então o controle de qualidade também na forma como se produzira o produto, ou seja, o processo de fabricação também sofrera inspeção sendo exigido melhorias continuas. $\mathrm{O}$ conceito de qualidade sofreu mais algumas alterações envolvendo os "custos da qualidade" e ainda o termo "defeito zero", objetivando a satisfação de todos os interessados.

O controle da qualidade se torna possível quando há um trabalho em conjunto, como mencionado anteriormente, pois o objetivo e alcançado com o desempenho de cada envolvido, tem como ponto central atingir um padrão de qualidade nos produtos a curto ou longo prazo sem perder sua originalidade, tomando ações e medidas no meio do processo produtivo, desde o processo de desenvolvimento, o processo fabril, estocagem, comercialização e pós-vendas.

\subsection{Qualidade no processo de desenvolvimento do produto}

Para garantir a qualidade do produto final, é necessário garantir a qualidade no início, ou seja, o produto não pode começar com falhas, levando em consideração a qualidade como um todo, é extremamente importante a exatidão na interpretação da necessidade do cliente, “conhecer para atender", nessa etapa ainda deve-se avaliar a manufaturabilidade do processo garantindo a execução do projeto com segurança e qualidade.

O processo e Desenvolvimento de Produto (PDP), caracteriza-se como uma série de procedimentos técnicos que parte na identificação da necessidade do cliente, ainda Rozenfeld (2006), há necessidade de avaliar e considerar as possibilidades e restrições tecnológicas da empresa, levando em consideração as estratégias competitivas de mercado e de produto.

No estudo em questão quanto à implantação, observou-se que em cada empresa possui suas dificuldades e quesitos passiveis de melhorias. Sendo assim afirma Bujis ( 2003), deve-se escolher o melhor modelo a ser utilizado de acordo com os objetivos e metas da empresa, 
complementando Goulart; Bernegozzi (2010), como a busca por melhorias é essencial para o sucesso organizacional, tornasse imprescindível a utilização de recursos e meios disponíveis no processo para obtenção com excelência de um produto adequado, avaliando sempre as possibilidades fabril, considerando a constante transformação da cultura focando em melhoria contínua.

\subsection{Qualidade no processo de fabricação}

O estudo revelou que o maior índice de não conformidades da empresa está na fabricação, sendo assim chegou-se à conclusão da utilização das ferramentas mais importantes do TQC ou Total Quality Control (Controle de Qualidade Total), que são: 5W2H, Diagrama de Pareto, Diagrama de causa e Efeito, Folha de verificação, Histograma, Gráfico de controle, Fluxograma e Brainstorming.

Folha de verificação: Essa ferramenta consiste em uma coleta de dados onde são identificados os problemas e defeitos causados no processo apresentados em planilhas ou "gráfico sarampo", os problemas mais frequentes, leva a uma identificação de problemas reais

Diagrama de Pareto: Consiste em um diagrama de barras onde classifica-se as ocorrências do maior para o menor acusando as ocorrências negativas de maior relevância.

Brainstorming: Essa ferramenta permite que os membros de um grupo discutem ideias, cada membro expõe sua ideia com o objetivo de juntos encontrarem uma solução para cada caso.

Diagrama de causa e efeito/ Ishikawa ou espinha de peixe: Esse diagrama tem o formato de uma espinha de peixe, seu objetivo e anotar o maior número de possíveis causas, analisando as entradas (imputs), o problema em questão fica na ponta e as demais causas nas setas laterais (espinhas), esse digrama é alimentado pelas informações adquiridas no Brainstorming.

Histograma: É um gráfico de colunas ou de barras que tem como objetivo representar a distribuição de frequência, mostrando as variações e oportunidades de ganhos.

Fluxograma: O objetivo dessa ferramenta e iniciar uma padronização objetivando a qualidade e aumentando a produtividade, com o mapeamento do processo.

Gráfico de controle: Esse gráfico limita o controle monitorando o processo mostrando os dados, permitindo isolar as anomalias devido as causas especiais isolando os resultados indesejáveis em problemas comuns, onde se utiliza o método PDCA. 
5W2H: É uma ferramenta simples onde o método consiste em definir o plano de ação organizacional, sendo claro e objetivo na ação a ser tomada e implantada. A aplicação dessa ferramenta exige sete perguntas importantes: What/ Que? Who/Quem? Were/ Onde? When/ Quando? Why/ Por que? How/ Como? How much/ Quanto?

O estudo feito entre os colaboradores mostra que os mesmos necessitam de treinamentos relacionados a qualidade, principalmente a conscientização da importância de trabalhar envolvidos nos processos pensando no processo seguinte, ainda a necessidade do sucesso do produto final depende de seguir criteriosamente os projetos desenvolvidos pela engenharia, ou seja seguir todos as especificações técnicas contidas nos desenhos, garantindo a qualidade do produto e a intercambialidade dos itens em caso de futura substituição por desgaste ou manutenção.

\subsection{Controle de máquinas operatrizes e ferramentas}

O estudo apontou a necessidade de abordar o tema controle de maquinas operatrizes e ferramentas. A qualidade de um produto usinado exige muito na condição de conservação do equipamento a ser utilizado, a precisão na geometria e na medida a ser alcançada, garantirá a montagem da peça no conjunto não dando margem para retrabalhos, e também a sua intercambialidade.

As ferramentas também deverão ser selecionadas de modo a dar condições ao operador, que por sua vez deverá ter habilidades para operar o equipamento e também identificar a ferramenta adequada para o processo, ferramentas desgastadas e inadequadas, além de colocar em risco a qualidade dimensional da peça, também prejudica a máquina causando esforços excessivos.

Para garantir a qualidade das maquinas operatrizes (MO), se faz necessário uma bateria de testes padronizados para avaliar se a máquina será capaz de usinar a peça respeitando todas as especificações técnicas exigidas pela engenharia, garantindo as tolerâncias e os desvios geométricos tolerados.

Ao efetuar o teste a máquina deverá estar livre de cargas, o próprio operador pode e deve executar periodicamente o teste ou se a necessidade exigir, um técnico especializado poderá acompanhar a verificação apontando os problemas que surgirem e tratando com urgência as devidas soluções. O teste consiste na verificação dos movimentos e superfícies da 


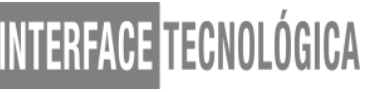

máquina que garantirão as tolerâncias geométricas e também os dimensionais das peças. Os fatores a serem verificados usualmente são:

- Geometria (paralelismo, retilineidade, perpendicularidade e cilindricidade)

- Desvio de posição das partes (localização, paralelismo e perpendicularidade entre eixos e superfícies).

Para verificação de cada item exigido no teste, será de extrema importância a utilização de instrumentos de precisão, documentar os valores das medidas encontradas comparando os dados. Para obter uma referência sobre a precisão exigida recomenda-se seguir as normas brasileiras descritas na MF-A5-00.00 do IPT, e ou nos manuais dos fabricantes (ISO 9001:2015-9.1).

\subsection{Controle dos Instrumentos de medição}

A garantia de se ter um parâmetro correto sobre a peça a ser usinada se dá através da utilização adequada por pessoal qualificado na utilização de instrumentos de medição. Cada dimensão ou cada geometria a ser avaliada poderá ou deverá ter o instrumento de medição especifico para garantir a precisão do resultado.

Assim como as maquinas operatrizes, os instrumentos também precisam passar por calibração periódica. Um instrumento de medição descalibrado, poderá colocar em risco todo o processo operacional.

A calibração de um instrumento objetiva avaliar o resultado do mesmo dentro de um intervalo aceitável, é feito uma comparação de uma medida padrão já conhecida e a medida obtida pelo instrumento, os dados de cada instrumento aferido, deverá ser armazenado, e de acordo com os respectivos históricos de calibração, poderá também acusar a necessidade do descarte do instrumento quando o mesmo não atender a precisão desejável (ISO 9001:20159.1).

\subsection{Rastreabilidade na indústria de maquinas e equipamentos}

A rastreabilidade é um fator importante, pois possibilita ter um registro onde contém todas as operações sofrida pelo produto alterando sua qualidade, pode também colaborar em 
levantamento de dados, onde as causas de defeitos posteriores ao processo, poderá ser avaliada e controlada com mais precisão.

O rastreio do produto tem como objetivo garantir um produto íntegro e de qualidade ao cliente através do controle de todas as etapas de cada processo. A rastreabilidade consiste em práticas sistêmica de separação dos itens, trocando as informações entre os envolvidos no processo, onde buscam o cumprimento de metas específicas, garantindo a qualidade dos produtos.

\footnotetext{
Rastreabilidade é o processo pelo qual se correlaciona de maneira clara e rápida, o lote de insumo com o do produto terminado, bem como, a localização do lote de produto terminado nos pontos de venda, tratando-se de produto alimentício (PELLEGRINI at al.,1996,p.2)
}

Capacidade de recuperar o histórico, a aplicação ou a localização daquilo que está sendo considerado (NBR ISSO 9000,2005,p.14).

A rastreabilidade é um complemento na gestão da qualidade que contribui em um conjunto de ações pela qualidade, portanto utilizada isoladamente não assegura o processo e o produto.

\subsection{Qualidade no prazo de entrega}

O prazo de entrega é de extrema relevância, tão importante quanto a entrega do produto acabado com qualidade, esse prazo é determinado no momento da venda do produto sendo assim, o cliente considera, ou seja, organiza seu processo produtivo contando com o novo equipamento adquirido, o não cumprimento do prazo pode prejudicar efetivamente seu cliente e ainda ferir sua marca e a confiança depositada pelo cliente.

Para garantir o prazo de entrega a empresa deverá fazer um levantamento dos produtos que serão possíveis manter em estoque, considerando os custos, esses produtos são aqueles que não podem faltar e são sempre utilizados. Uma gestão eficaz do estoque pode ser muito útil na hora de realizar os processos evitando surpresas na hora de realizar as tarefas.

Uma relação de confiança com os fornecedores garante a entrega da mercadoria dentro do prazo, uma boa equipe de compradores pode levantar fornecedores confiáveis fazendo pesquisas, entrando em contato com outras empresas, buscando referencias de parcerias e a qualidade do produto. 
Muito importante que a empresa mantenha uma comunicação clara com seu cliente, informando cada etapa do processo (cronograma), e sendo claro e sincero ao surgir problemas que acarretara o não cumprimento do prazo.

\subsection{Qualidade do produto acabado}

O produto acabado em questão, trata-se de maquinas hidráulicas de alta precisão, que por sua vez fará parte do processo de fabricação do cliente, como mencionado acima, é de extrema importância garantir a geometria do equipamento, a precisão de parada e a capacidade de prensagem. Para garantir a geometria, a matéria prima mais especificamente o aço que será utilizado na estrutura da máquina, junto com o processo de soldagem e usinagem, deverá atender todas as especificações exigidas pela engenharia a fim de garantir a integridade estrutural da máquina.

A precisão da força e do movimento se dá através dos componentes hidráulicos e eletrônicos, que deverão ser selecionados pela engenharia a fim de atender a necessidade do cliente exigida no início do processo de desenvolvimento. Esses componentes assim como todo o equipamento, deverão atender as exigências da norma regulamentadora NR 12.

Após a conclusão da montagem do equipamento, o mesmo deverá passar por uma bateria de teste onde serão avaliados todos os itens de segurança, precisão de movimento e capacidade de prensagem. O cliente por sua vez sempre que possível, ou se a ocasião exigir, fornecerá toda condição necessária para que seu processo seja simulado e testado antes da entrega do equipamento, o mesmo acompanhará os resultados do teste e se houver alguma anomalia, deverá ser resolvido sem prejudicar o prazo de entrega.

\subsection{Qualidade no pós-venda}

Umas das tarefas mais difíceis é a fidelização do cliente, mesmo porque, como aponta no início desse artigo, há uma concorrência acirrada no mercado industrial de maquinas e equipamentos, mas especificamente máquinas hidráulicas, em vista disso deve-se atentar em realizar um pós-venda de qualidade, atendendo o cliente prontamente, garantindo ainda mais sua satisfação e o mesmo retorne fazendo novos negócios com a empresa, isso dará início a uma fidelização. Um pós-venda efetivo, pode ser o diferencial competitivo principalmente no 
mercado de máquinas especificas atendendo um processo especifico, gerando oportunidade do cliente promover indicações da empresa e do produto ajudando na hora de fechar um novo negócio.

\section{PROPOSTA DE MELHORIA COM O USO DA GESTÃo PELA QUALIDAdE TOTAL}

Esse estudo propõe durante a aplicação da Gestão pela qualidade total, a utilização de checklist contendo todos os requisitos pertinentes a cada processo de inspeção assegurando o acompanhamento de cada item especificadamente.

O estudo ainda propõe a orientação dos órgãos competentes para a certificação da empresa, que fará com que o objetivo alcançado permaneça, garantindo um padrão de qualidade sólido. A ISO 9000 é um conjunto de normas e diretrizes internacionais reconhecida mundialmente que dará suporte na gestão da qualidade, na melhoria contínua aumentando a eficiência da empresa atingindo os objetivos e metas de permanecer no mercado com destaque.

A gestão da qualidade total não é uma tarefa fácil, pois existem dificuldades a serem vencidas e obstáculos a serem superados, mas no momento em que há uma estratégia, ou seja, uma mudança de comportamento visando a qualidade, o objetivo pela qualidade total ficará mais tangível, ou melhor mais próximo da realização, pois a qualidade é um alicerce para um negócio rentável.

\section{RESULTADOS E DISCUSSÃO}

O estudo de caso escolhido apresenta resultados que mostram a extrema importância na aplicação das ferramentas de gestão pela qualidade total, pois é uma das mais importantes metas que as maiores organizações buscam atingir, pois garantir a satisfação dos clientes e dos interessados (stakeholders), torna-se vital para a permanência no mercado de maquinas e equipamentos hidráulicos, além de existir metodologias no caso da gestão pela qualidade total envolvendo TQC ou Total Quality Control (Controle de Qualidade Total), que auxilia na melhoria contínua alcançando a excelência dos processos de desenvolvimento até a entrega do equipamento testado e aprovado seguindo rigorosas normas internas de qualidade.

Após a implantação dos métodos de melhorias sugeridas no estudo de caso em questão, foi possível entender e atender os principais problemas de qualidade em cada área 
envolvida, onde foi possível a avaliação de cada problema possibilitando uma tomada de decisão efetiva buscando maiores ganhos e competitividade.

\section{CONSIDERAÇÕES FINAIS}

O estudo mostrou que a aplicação da gestão pela qualidade total na fabricação de prensas hidráulica se fez de suma importância satisfazendo esse mercado industrial extremamente competitivo, pois a organização que melhor dominar esse conceito, terá vantagem comercial com um produto de qualidade e custo competitivo.

A concorrência no mercado industrial em todo mundo exige uma busca pela melhoria, para obter uma posição de destaque, as empresas utilizam métodos, metodologias e estudos visando a qualidade total de seus produtos e serviços, objetivando também certificações que assegurará a seus clientes o compromisso da organização com a qualidade em cada processo produtivo.

A Gestão da qualidade, é formada por uma equipe estratégica da empresa que préestabelece padrões, metodologias e procedimentos visando a satisfação dos clientes e stakeholders. Os integrantes da equipe estratégica (Gestores da qualidade), da empresa deve estar sempre alerta, verificando com frequência o compromisso ou melhor, o envolvimento dos colaboradores em cada etapa do processo, onde cada passo à frente conquistado não seja frustrado por erros e insatisfação por menor que seja do cliente.

\section{REFERÊNCIAS}

CASTRO, A. et al. ISO: uma ferramenta para a garantia da qualidade no processo produtivo, artigo apresentado no I Encontro Científico e Simpósio de Educação Unisalesiano, 17 a 20 de outubro,

DELIC, M.; RADLOVACK, V.; KAMBEROVIC, B.; MAKSIMOVIC R.; PECUJLIJA, M. Examinng relationships between quality management and organizational performance in transitional economies. Total Quality Management \& Business Excellence, v.25, n. 3-4, p. 367-382,10 Fev 2014. 
RACHID, A. Participação dos trabalhadores na Gestão da Produção. Revista Gestão \& Conexões, v. 2, n.2, 15 Nov 2013. Disponível em:

http://www.periodicos.ufes.br/ppgadm/article/view/4678>. Acesso em: 20 maio 2016.

SCHEIN, E. H. Cultura organizacional e liderança. São Paulo: Atlas, 2009

ROZENFELD, H. et al. Gestão de desenvolvimento de produtos - uma referência para a melhoria do processo. São Paulo: Saraiva, 2006.

BUJIS, J.. Modelling Product Innovation Processes, from Linear Logic to Circular Chaos. Creativity and innovation management, v. 12, n. 2, 2003.

GOULART, L. E T., BERNEGOZZI, R. P. O uso das ferramentas da Qualidade na melhoria de processos produtivos. In: INTERNATIONAL CONFERENCE ON INDUSTRIAL ENGINEERING AND OPERATIONS MANAGEMENT, 16., 2010, São Carlos. Anais...São Carlos, 2010.

DAHLGAARD, J. J.; CHEN, C.; JANG, J.; BANEGAS, L. A.; DAHLGAARD-PARK S. M. Business excellence models: limitations, reflections and further development. Total Quality Management \& Business Excellence, v. 24, n. 5-6, p. 519-538, Jun 2013.

GREEN, T. J. TQM and organisational culture: How do they link? Total Quality Management \& Business Excellence, v. 23, n. 2, p. 141-157, Fev 2012.

PELLEGRINI, F. T.; GALHARDI, M.G.; CASTRO, R.L.G. Rastreabilidade de insumos e Produtos para empresas de Alimentos. Campinas, SP: SBCTA, 1996.

GIMENEZ-ESPIN, J. A.; JIMÉNEZ-JIMÉNEZ, D.; MARTÍNEZ-COSTA, M. Organizational culture for total quality management. Total Quality Management \& Business Excellence, v. 24, n. 5-6, p. 678-692, Jun 2013.

GAMBI, L. N.; BOER, H.; GEROLAMO, M. C.; JORGENSEN, F.; CARPINETTI, L. C. R. The relationship between organizational culture and quality techniques, and its impact on operational performance. International Journal of Operations \& Production Management, v. 35, n. 10, p. 1460-1484, 5 Out 2015.

HAFFAR, M.; AL-KARAGHOULI, W.; GHONEIM, A. The mediating effect of individual readiness for change in the relationship between organisational culture and TQM implementation. Total Quality Management \& Business Excellence, v. 24, n. 5-6, p. 693706, Jun 2013.

Lins/Brasil, 2007. Disponível em: http://www.unisalesiano.edu.br/encontro2007/ > Acesso em 12 abr. 2009

Controle da qualidade total disponível em:

<https://www.infoescola.com/administracao_/controle-de-qualidade-total-tqc/>. Acesso em: 24 jul. 2019. 
Testes de maquinas operatrizes. Disponível em:

<http://www.mecanicaufrj.educacao.ws/util/b2evolution/media/blogs/flavio/TesteMaquinas20 120627.pdf>. Acesso em: 30 jul. 2019

Prazo de entrega satisfação do cliente. Disponível em:

https://cargox.com.br/blog/como-garantir-o-prazo-de-entrega-e-a-satisfacao-do-cliente Acesso em: 30 jul. 2019

A importância de um pós-venda de qualidade. Disponível em:

$<$ https://blog.softium.com.br/index.php/descubra-importancia-de-um-pos-venda-dequalidade/>. Acesso em: 31 jul. 2019

O que é gestão da qualidade total - disponível em:

<http://gestao-de-qualidade.info/qualidade-total.html> Acesso em: 25 jul. 2019 